\title{
FROM ENVIRONMENTAL FLUCTUATIONS AND ENERGY AVAILABILITY TO EVOLUTIONARY CHANGE AND SPECIATION
}

PARSONS, Peter A., Waite Institute, University of Adelaide, Glen Osmond, SA 5064, Australia.

Evolutionary patterns can be considered in terms of two interacting continuums (a) the magnitude of environmental fluctuations of physical variables especially climatic, and (b) the availability of metabolic energy beyond basic needs for maintenance and survival (Parsons, 1991) so giving four extreme combinations:

(1) widely fluctuating environments, low metabolic energy availability. This situation is a feature of species borders where the energetic costs of environmental perturbations restrict normal physiological processes so that ecological range expansions are precluded. Provided extinctions do not occur, species would alter their ranges to track longer term climatic changes as described in the historical and fossil records.

(2) widely fluctuating environments, high metabolic energy availability. This combination favors opportunists and colonists. Furthermore, following a relaxation of stress intensity after a mass extinction there could be a burst of speciation in such generalist species.

(3) stable environments, low metabolic energy availabilty. This combination represents deep sea and cave environments where low metabolic rate is an adaptation to accomodate stresses such as anoxia and low resource availability. Consequently the capacity for evolutionary change is low in these habitats which tend to contain relict species.

(4) stable environments, high metabolic energy availability, On energetic grounds speciation is theoretically possible, but high vulnerability to any stressful perturbation would be restrictive in practice since some stress is the norm in natural populations.

The potential for evolutionary change and speciation is low in these four extremes, however in intermediate habitats the potential is higher as implied in (2) when stress intensity is relaxed. The intermediate habitats would be characterized by moderately fluctuating environments where the metabolic cost of stress would not preclude adaptive change. Furthermore, the stress levels in such habitats should lead to sufficient underlying genetic variability to be permissive of adaptive responses to environmental change (Parsons, 1991). This paradigm may apply to speciation in tropical rain forests. A fossil analogy would be marine invertebrates in frequently disturbed onshore habitats.

PARSONS, P.A. 1991. Evolutionary rates: stress and species boundaries. Annual Review of Ecology and Systematics 22:1-18. 